\title{
PROPERTIES OF THERMAL GLUEBALLS*
}

\author{
NORIYOSHI ISHII \\ Radiation Laboratory, \\ RIKEN (The Institute of Physical and Chemical Research), \\ 2-1 Hirosawa, Wako, Saitama 351-0198, Japan \\ E-mail: ishii@rarfaxp.riken.go.jp \\ HIDEO SUGANUMA \\ Faculty of Science, Tokyo Institute of Technology, \\ 2-12-1 Ohokayama, meguro, Tokyo 152-8550, Japan
}

\begin{abstract}
We study the properties of the $0^{++}$glueball at finite temperature using $\mathrm{SU}(3)$ quenched lattice QCD. We find a significant thermal effects near $T_{c}$. We perform the $\chi^{2}$ fit analyses adopting two Ansätze for the spectral function, i.e., the conventional narrow-peak Ansatz and an advanced Breit-Wigner Ansatz. The latter is an extension of the former, taking account of the appearance of the thermal width at $T>0$. We also perform the MEM analysis. These analyses indicate that the thermal effect on the glueball is a significant thermal-width broadening $\Gamma\left(T_{c}\right) \sim 300$ $\mathrm{MeV}$ together with a modest reduction in the peak center $\Delta \omega_{0}\left(T_{c}\right) \sim 100 \mathrm{MeV}$.
\end{abstract}

Near the critical temperature $T_{c}$, the QCD vacuum begins to change its structure. For instance, the string tension reduces and the spontaneously broken chiral symmetry begins to restore partially. Since hadrons are composites of quarks and gluons, whose interaction depends on the properties of the QCD vacuum, it is natural to expect the structural changes of various hadrons. In this direction, there are a number of studies based on QCD motivated effective models, which predict the mass reductions of charmonium and light $q \bar{q}$ mesons near $T_{c} .{ }^{1,2}$ In BNL-RHIC experiments, these changes are considered as important precritical phenomena of QCD phase transition. In addition, lattice QCD Monte Carlo calculations have been performed to examine the pole-mass shifts of hadrons at the quenched level. ${ }^{3,4}$ The $0^{++}$glueball is one of such hadrons, which are expected to ex-

*The numerical calculation has been done on NEC SX-5 at Osaka Univeristy. 
hibit structural changes near $T_{c}$. Studies based on the effective models ${ }^{5,6}$ suggest a large mass reduction of the glueball as a natural consequence of the sizable difference between the $0^{++}$glueball mass $m_{\mathrm{G}}=1500-1700$ $\mathrm{MeV}$ and the critical temperature $T_{c}=260-280 \mathrm{MeV}$. In this paper, we investigate the glueball properties at finite temperature by means of lattice QCD Monte Carlo calculation. ${ }^{7,8,9}$

We consider the temporal correlator $G(\tau) \equiv\langle\phi(\tau) \phi(0)\rangle$ of the glueball operator $\phi(\tau)$. The simplest construction of the glueball operator is the plaquette operator, which, however, is known to have only a negligible overlap to the lowest-lying $0^{++}$glueball. To enhance the overlap, the smearing method provides us with a systematic procedure by extending the spatial size of the operator. In this paper, we adopt the APE smearing. ${ }^{8,10}$ The continuum expression of the smeared glueball operator is obtained in the Coulomb gauge as

$$
\phi \propto \int \frac{d^{3} y d^{3} z}{(2 \pi)^{3 / 2} \rho^{3}} \exp \left(-\frac{(\vec{y}-\vec{z})^{2}}{2 \rho^{2}}\right) G_{i j}^{a}(\vec{y}) G_{i j}^{a}(\vec{z}),
$$

where $G_{i j}^{a}$ is the field strength tensor, and $\rho \in \mathbb{R}$ controls the size of the spatial extension. (For detail, see Refs. 8.) By choosing $\rho$ appropriately, we can maximize the contribution from the lowest-lying glueball in the glueball correlator $G(\tau)$.

We next consider the spectral representation of $G(\tau) \equiv\langle\phi(\tau) \phi(0)\rangle$ as

$$
G(\tau)=\int_{0}^{\infty} d \omega K(\tau, \omega) A(\omega)
$$

with $\beta \equiv 1 / T, K(\tau, \omega) \equiv \frac{\cosh (\omega(\beta / 2-\tau))}{\sinh (\beta \omega / 2)}$, and $A(\omega)$ denotes the spectral function with its spatial momentum projected to zero. At low temperature, peak positions of $A(\omega)$ provide us with the pole masses of hadrons. Hence the simplest parameterization of $A(\omega)$ for the $\chi^{2}$ fit analysis is given as ${ }^{7}$

$$
A(\omega)=C\{\delta(\omega-m)-\delta(\omega+m)\},
$$

where $C$ and $m$ are used as the fit parameters, corresponding to the strength and the pole mass, respectively. To adopt this Ansatz, the peak should be narrow enough. We will refer to Eq. (3) as the "narrow-peak Ansatz". At $T>0$, however, through the interaction with the thermally excited particles, the thermal width is generated even for stable particles. As $T$ gets larger, the effects of the thermal width become more important. To respect its existence, we adopt the following parameterization $a^{8}$

$$
A(\omega)=C\left\{\delta_{\Gamma}\left(\omega-\omega_{0}\right)-\delta_{\Gamma}\left(\omega+\omega_{0}\right)\right\},
$$


where $\delta_{\Gamma}(\omega) \equiv \frac{1}{\pi} \operatorname{Im}\left(\frac{1}{\omega-i \Gamma}\right) . C, \omega_{0}$, and $\Gamma$ are used as the fit parameters corresponding to the strength, the peak center, and the thermal width, respectively. We will refer to Eq. (4) as the "Breit-Wigner Ansatz". Note that $\lim _{\Gamma \rightarrow 0} \delta_{\Gamma}(\omega)=\delta(\omega)$. Hence, the Breit-Wigner Ansatz is a natural extension of the narrow-peak Ansatz.

For these $\chi^{2}$ fit analyses, proper Ansätze for $A(\omega)$ have to be provided. However, in the very vicinity of $T_{c}$, and above $T_{c}$, there may appear more complicated structures. In this sense, it is desirable to obtain $A(\omega)$ directly from $G(\tau)$, which, however, is known to be an ill-posed problem numerically. Recently developed Maximum Entropy Method (MEM) ${ }^{11,12}$ can deal with this inverse problem adopting the following Shannon-Jaynes entropy as

$$
S \equiv \int_{0}^{\infty} d \omega\left[A(\omega)-m(\omega)-A(\omega) \log \left(\frac{A(\omega)}{m(\omega)}\right)\right]
$$

where $m(\omega)$ is a real and positive function referred to as the default model function. $m(\omega)$ is required to reproduce the asymptotic behavior of $A(\omega)$ as $\omega \rightarrow \infty$.

We use the $\mathrm{SU}(3)$ anisotropic lattice plaquette action $^{13}$ as

$$
S_{\mathrm{G}}=\frac{\beta_{\text {lat }}}{N_{c} \gamma_{\mathrm{G}}} \sum_{s, i<j \leq 3} \operatorname{Re} \operatorname{Tr}\left\{1-P_{i j}(s)\right\}+\frac{\beta_{\text {lat }} \gamma_{\mathrm{G}}}{N_{c}} \sum_{s, i \leq 3} \operatorname{Re} \operatorname{Tr}\left\{1-P_{i 4}(s)\right\},
$$

where $P_{\mu \nu}(s) \in \mathrm{SU}(3)$ denotes the plaquette operator in the $\mu$ - $\nu$-plane. The lattice parameter and the bare anisotropic parameter are fixed as $\beta_{\text {lat }} \equiv 2 N_{c} / g^{2}=6.25$ and $\gamma_{\mathrm{G}}=3.2552$, respectively, so as to reproduce the renormalized anisotropy as $a_{s} / a_{t}=4$. The scale unit is introduced from the on-axis data of the static inter-quark potential with the string tension $\sqrt{\sigma}=440 \mathrm{MeV}$. The resulting lattice spacings are given as $a_{t}^{-1}=9.365(66)$ $\mathrm{GeV}$ and $a_{s}^{-1}=2.341(16) \mathrm{GeV}$. The critical temperature is estimated as $T_{c} \simeq 280 \mathrm{MeV}$ from the susceptibility of the Polyakov loop. ${ }^{8}$ We generate 5,500-9,900 gauge configurations to construct the glueball correlators, ${ }^{7,8}$ where statistical data are divided into bins of the size 100 to reduce possible auto-correlations near $T_{c}$. To enhance the low-energy spectrum, we adopt an appropriate smearing corresponding to $\rho \simeq 0.4 \mathrm{fm}$ in Eq. (1).

We present the numerical results. Whereas the narrow-peak analysis suggests that the thermal effect on the glueball is the pole-mass reduction ${ }^{7}$ of about $300 \mathrm{MeV}$, the advanced Breit-Wigner analysis suggests that it is a thermal-width broadening ${ }^{8}$ of about $300 \mathrm{MeV}$ together with a modest reduction in the peak center of about $100 \mathrm{MeV}$. The numerical results of 
the $\chi^{2}$ fit analyses with the Breit-Wigner Ansatz are shown in Fig. 1.

$\omega_{0}[\mathrm{MeV}]$

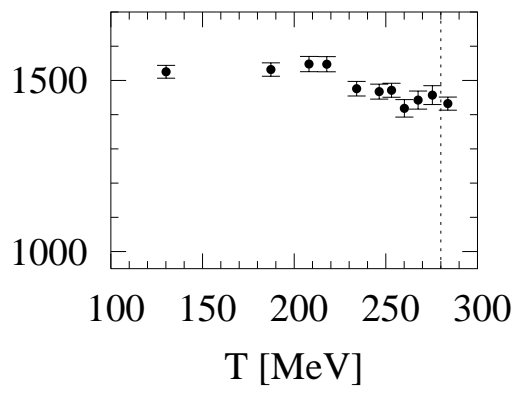

$\Gamma[\mathrm{MeV}]$

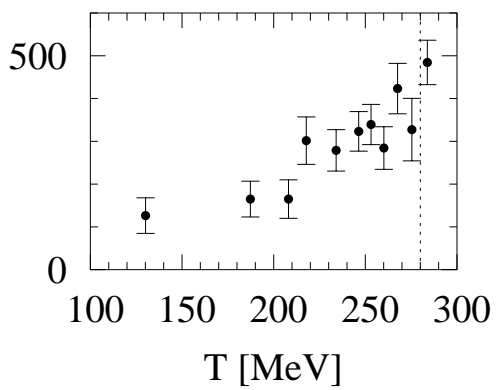

Figure 1. The peak center $\omega_{0}(T)$ and the thermal width $\Gamma(T)$ of the $0^{++}$glueball against temperature $T$, obtained from the $\chi^{2}$ fit analysis with the Breit-Wigner Ansatz. The vertical dotted lines indicate the critical temperature $T_{c} \simeq 280 \mathrm{MeV}$.

We now turn to the result of MEM analysis. As the default model function, we adopted the perturbative expression of the smeared glueball correlator up to $O\left(\alpha_{\mathrm{S}}^{0}\right)$ as $^{9}$

$$
m(\omega)=N \omega^{4} \exp \left\{-(\omega \rho)^{2} / 4\right\},
$$

where the normalization factor $N$ is determined from $1=\int_{0}^{\infty} d \omega K(\tau=$ $0, \omega) m(\omega)$ so as to mimic $G(\tau=0)=1$, which we adopt as the normalization of the correlator. Following the numerical procedure given in Ref. 12, we reconstruct $A(\omega)$. The results at various temperatures are shown in Fig. 2. We see that the peak tends to become broader with the increasing temperature,${ }^{9}$ which is consistent with the results of the Breit-Wigner fit analysis.

To summarize, we have studied the properties of thermal glueballs below $T_{c}$ by using SU(3) anisotropic lattice QCD at the quenched level. We have performed $\chi^{2}$ fit analyses adopting two Ansätze for the spectral function $A(\omega)$, i.e., the conventional narrow-peak Ansatz ( $\delta$-function type) and the advanced Breit-Wigner Ansatz so as to respect the appearance of the thermal width at $T>0$. Whereas the former has suggested that a significant pole mass reduction of the $0^{++}$glueball of about $300 \mathrm{MeV}$ near $T_{c}$, the latter has suggested that the thermal effects on the glueball is rather a significant thermal-width broadening of about $300 \mathrm{MeV}$ together with a modest reduction in peak center of about $100 \mathrm{MeV}$. We have also performed MEM analysis. We have seen that the peak of the $0^{++}$glueball tends to 


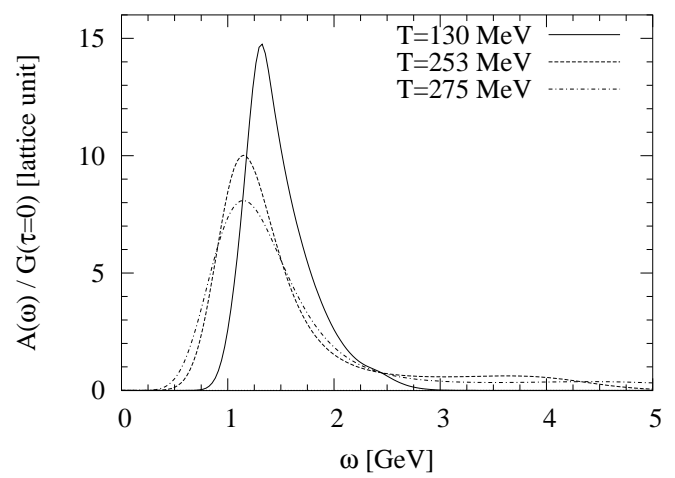

Figure 2. MEM results for the $0^{++}$glueball spectrum at various temperatures below $T_{c}$.

become broader with the increasing temperature below $T_{c}$. This is consistent with the results of the advanced Breit-Wigner fit analysis, suggesting the importance of the thermal width. Experimentally, there are two glueball candidates, ${ }^{14}$ i.e., $f_{0}(1500)$ and $f_{0}(1710)$, both of which have rather narrow decay width $\Gamma \simeq 100 \mathrm{MeV}$. Hence, the experimental observation of thermal-width broadening of $300 \mathrm{MeV}$ would be possible in the future experiments at RHIC. For more realistic comparison with experiments, it is desirable to estimate the effects of the dynamical quarks on the thermal properties of the glueball.

\section{References}

1. T. Hashimoto, K. Hirose, T. Kanki and O. Miyamura, Phys. Rev. Lett. 57, 2123 (1986).

2. T. Hatsuda and T. Kunihiro, Phys. Rev. Lett. 55, 158 (1985).

3. QCD-TARO Collaboration, Phys. Rev. D63, 054501 (2001).

4. T. Umeda, R. Katayama, O. Miyamura and H. Matsufuru, Int. J. Mod. Phys. A16, 2215 (2001).

5. H. Ichie, H. Suganuma and H. Toki, Phys. Rev. D52, 2944 (1995).

6. N. Ishii and H. Suganuma, Eur. Phys. J. A17, 77 (2003).

7. N. Ishii, H. Suganuma and H. Matsufuru, Phys. Rev. D66, 014507 (2002).

8. N. Ishii, H. Suganuma and H. Matsufuru, Phys. Rev. D66, 094506 (2002).

9. N. Ishii and H. Suganuma, Nucl. Phys. B(Proc. Suppl), hep-lat/0309102.

10. APE Collaboration, Phys. Lett. B192, 163 (1987).

11. M. Jarrell and J.E. Gubernatis, Phys. Rept. 269, 133 (1996).

12. M. Asakawa, T. Hatsuda and Y. Nakahara, Prog. Part. Nucl. Phys. 46, 459 (2001).

13. T. R. Klassen, Nucl. Phys. B533, 557 (1998).

14. K.K. Seth, Nucl. Phys. A675, 25c (2000). 